\title{
BMJ Open Efficacy of dehydroepiandrosterone to overcome the effect of ovarian ageing (DITTO): a proof of principle randomised controlled trial protocol
}

\author{
Kannamannadiar Jayaprakasan, ${ }^{1,2,3}$ Amarin Narkwichean, ${ }^{1}$ Walid E Maalouf, ${ }^{1}$ \\ Bruce K Campbell ${ }^{1}$
}

To cite: Jayaprakasan $\mathrm{K}$, Narkwichean A, Maalouf WE, et al. Efficacy of dehydroepiandrosterone to overcome the effect of ovarian ageing (DITTO): a proof of principle randomised controlled trial protocol. BMJ Open 2014;4:e005767. doi:10.1136/bmjopen-2014005767

- Prepublication history for this paper is available online To view these files please visit the journal online (http://dx.doi.org/10.1136/ bmjopen-2014-005767)

Received 23 May 2014 Revised 4 September 2014 Accepted 5 September 2014

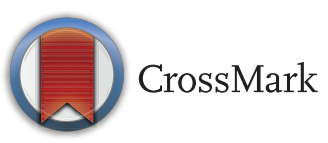

${ }^{1}$ Division of Child Health, Obstetrics and Gynaecology, School of Medicine, Faculty of Medicine and Health Sciences, University of Nottingham, Nottingham, UK ${ }^{2}$ Nottingham University Research and Treatment Unit in Reproduction (NURTURE), Queen's Medical Centre, University of Nottingham, Nottingham, UK

${ }^{3}$ Derby Fertility Unit, Royal Derby Hospital, Derby, UK

Correspondence to Dr Kannamannadiar Jayaprakasan;

k.jayaprakasan@nottingham. ac.uk

\section{ABSTRACT}

Introduction: Dehydroepiandrosterone (DHEA) has been proposed to improve pregnancy rates in women with diminished ovarian reserve undergoing in vitro fertilisation (IVF) treatment. However, evidence regarding its efficacy is supported by a limited number of randomised controlled trials (RCTs). This doubleblinded RCT aims to measure the effect of DHEA supplementation prior to and during controlled ovarian hyperstimulation on ovarian response prior to IVF treatment in women predicted to have poor ovarian reserve.

Methods and analysis: Sixty women with ovarian antral follicle count $\leq 10$ and serum anti-Mullerian hormone $\leq 5 \mathrm{pmol} / \mathrm{L}$ undergoing IVF/intracytoplasmic sperm injection (ICSI) treatment at the Nurture fertility clinic, Nottingham will be recruited. They will be randomised to either receive DHEA capsule $75 \mathrm{mg} /$ day or placebo for at least 12 weeks before egg collection. All participants will undergo standard long down regulation protocol using human menopausal gonadotropin $300 \mathrm{IU} /$ day. Serum samples and follicular fluids at the time of egg collection will be collected for hormonal immunoassays. For ICSI participants, cumulus cells stripped from oocyte will be collected for cumulus gene expression analyses regarding oocyte competence. Microdrops of oocyte culture media before the time of ICSI will be assessed for glucose, pyruvate and lactate utilisation. Embryo transfer will be performed on day 2, 3 or 5 based on the number and quality of the embryos available. Pregnancy will be defined as urine pregnancy test positive (biochemical pregnancy) and 6-8 weeks ultrasound scan with fetal heart beat (clinical pregnancy) and live birth. It is planned to perform the molecular and nutritional fingerprint analyses in batches after finishing the clinical phase of the study.

Ethics and dissemination: The approval of the study was granted by the NHS Research Ethics Committee (Ref number NRES 12/EM/0002), the Medicines and Healthcare products Regulatory Agency (MHRA), and the Nottingham University Hospitals Trust Research and Development department. All participants shall provide written informed consent before being randomised into allocated treatment groups.

\section{Strengths and limitations of this study}

- The study is a double-blinded placebo-controlled randomised controlled trial. Randomisation numbers will be generated by the computer system, and therefore the patient and the healthcare team will not know which treatment (dehydroepiandrosterone (DHEA) or placebo) the patient receives.

- The authors also plan to use molecular studies of the cumulus gene expression and media nutritional fingerprint to elucidate the mechanism of DHEA on the oocyte development.

- As this is a pilot study, the intended sample size is small and plans to recruit only 60 participants.

Trial registration number: Protocol V.2.0; EudraCT number: 2011-002425-21; http://www.clinicaltrials.gov; NCT01572025; CTA reference: 03057/0053/001-0002

\section{INTRODUCTION}

One of the major changes in the societies noted worldwide over the past few decades is postponement of childbearing due to women pursuing higher education and a successful career. Currently, large numbers of women defer attempting to conceive until their mid-30s or 40s. ${ }^{1}$ For instance, in the UK, there has been a rise in childlessness at the age of 35 from $12 \%$ for those born in 1941 to $25 \%$ for those born in $1971 .^{2}$ An important issue that has arisen consequently from this trend is the marked increase in the incidence of women with infertility who seek medical interventions to overcome the involuntary childlessness incurred as a result of ovarian ageing.

Ovarian ageing, dictated by a decline in the quantity and quality of oocytes within the ovaries, ${ }^{3}{ }^{4}$ is responsible for the wellestablished observation of age-related decline 
in fertility $^{5-8}$ and of age-related increase in adverse reproductive events such as miscarriages ${ }^{9}{ }^{10}$ and aneuploid pregnancies. ${ }^{11-13}$ While the age-related decline in fertility cannot be overcome with conventional in vitro fertilisation (IVF) treatment, reduced ovarian reserve secondary to ovarian ageing is one of the major factors determining the success of IVF outcome. Further, loss of ovarian function can have additional health consequences through effects on metabolism, cardiovascular function, cognition, response to stress, bone strength ${ }^{14-}$ ${ }^{16}$ and risk of malignancies. ${ }^{17}$

While chronological age is an important determinant of ovarian ageing, there is a considerable interindividual variation in the rate of the ageing process, possibly influenced by genetic and environmental factors. The pace at which ovarian ageing occurs is determined by the rate of primordial follicle initiation, the rate of follicle turnover or loss and the rate of follicle and oocyte maturation. ${ }^{18} 19$ These processes are regulated by intrafollicular and interfollicular interactions mediated by pituitary derived gonadotropins and oocytes and somatic cell-derived local growth regulators, which may be liable to therapeutic manipulation with drugs such as dehydroepiandrosterone (DHEA).

DHEA is a weak androgenic steroid secreted mainly from the adrenal glands but also from ovaries and by peripheral conversion. The serum levels of DHEA decline markedly with advancing age. It has been proposed that the oral administration of DHEA in women may have antiageing effects and improve well-being and sexual function. A few observational studies reported that DHEA improves ovarian response and pregnancy rates in women with reduced ovarian reserve following assisted reproduction treatment (ART). ${ }^{20-26}$ In one of the only three randomised controlled trials (RCTs) published so far, a group of 33 women with reduced ovarian reserve were randomised to receive either $75 \mathrm{mg}$ DHEA per day orally for at least 6 weeks before starting the first cycle of ovulation induction or no pretreatment. Participants who did not conceive and continued to the second cycle took DHEA for at least 16 weeks, while the average duration of DHEA intake by the study group was 13.5 weeks. While there was no significant difference in terms of mean number of retrieved oocytes and fertilisation rates, the cumulative live birth rates over two IVF cycles $(23.1 \%$ vs $4 \%$; $\mathrm{p}=0.05)$ were significantly higher in the DHEA group compared with the control group. ${ }^{26}$ It was suggested that the benefit of DHEA treatment would be most effective if it was supplemented for at least 3 months, which is equal to the time needed to affect the gonadotropin-responsive follicle pool. Therefore, they believed that DHEA acts in ovarian recruitment and early folliculogenesis. ${ }^{27}$ However, there were few methodological issues with that trial. It was not blinded. It was registered at the ClinicalTrials.gov as controlled clinical trial (CCT) on 3 May 2010 after the trial was completed in July 2009, in fact after the trial report had been submitted for publication in February 2012.
The authors freely admitted that they had no prespecified sample size or primary end point, and were inspecting the results as data accumulated and stopped when they achieved a nominally significant difference in pregnancy rate.

While subsequent RCTs failed to demonstrate significant improvement after DHEA supplementation, ${ }^{28} 29$ the other controlled studies reported in the literature so far suggest that DHEA may improve quantitative and qualitative ovarian response. It is important to note the weak methods as they were not blinded and employed only small sample sizes. ${ }^{30}$ Further investigation into the role of DHEA on ovarian ageing is required and women undergoing ART provide an ideal model with whom we can evaluate the developmental competence of oocytes both in vivo and in vitro. Hence, this warrants a welldesigned and large RCT to examine the effect of DHEA in women with diminished ovarian reserve undergoing ART treatments. While there are many ovarian reserve tests with varying predictive abilities reported, antral follicle count (AFC) and anti-Mullerian hormone (AMH) have consistently been found to have the best diagnostic accuracy to predict poor ovarian reserve. ${ }^{31}$

We are proposing a pilot randomised, double-blinded, placebo controlled trial to examine the feasibility of conducting a large trial, in addition to exploring the effect of DHEA on oocyte quantity and quality. The principal objective of this study is to evaluate the effect of DHEA on the ovarian response to gonadotropins (oocyte quantity) during controlled ovarian stimulation and the developmental competence of oocytes (oocyte quality) by using molecular and clinical markers in women with aged ovaries, identified by using reported AFC and AMH thresholds. ${ }^{32-34}$

\section{MATERIALS AND METHODS}

\section{Design, setting and participants}

This study is planned as a double-blind, placebocontrolled, randomised pilot trial, being conducted at a university-based tertiary fertility centre (Nottingham University Research and Treatment Unit in Reproduction (NURTURE), University of Nottingham, UK). An expected number of 60 patients undergoing either IVF or intracytoplasmic sperm injection (ICSI) and predicted to have poor ovarian response will be recruited to this RCT. The study participants will receive oral capsules of either $75 \mathrm{mg}$ of DHEA or placebo for at least 12 weeks before starting ovarian stimulation with gonadotropin during IVF/ICSI treatment. Participants will be provided with a maximum 20-week supply of medication. A DHEA capsule is made from active $75 \mathrm{mg}$ DHEA powder encapsulated in the white gelatine capsule by the licensed pharmaceutical company authorised by the Medicines and Healthcare products Regulatory Agency (MHRA). Placebo is also produced from the capsule with similar size, colour and appearance but without the DHEA powder. 


\section{Inclusion criteria}

1. Women aged 23-43 years with diminished ovarian reserve (predicted to be poor responders), defined as AFG scan $\leq 10$ and/or serum AMH $\leq 5 \mathrm{pmol} / \mathrm{L}$ undergoing IVF or ICSI treatment.

2. Women must have a regular spontaneous menstrual cycle of 21-35 days.

3. Women must be willing and comply with scheduled visits, the treatment plan and laboratory tests and give written informed consent.

\section{Exclusion criteria}

1. Women with body mass index $>35 \mathrm{~kg} / \mathrm{m}^{2}$;

2. Women with a single ovary;

3. Women with untreated hydrosalpinx/submucous fibroid/endometrial polyp at the start of treatment;

4. Women with any history of seizure disorders;

5. Women with previous participation in this trial in an earlier treatment cycle;

6. Women with any known endocrine disorders such as congenital adrenal hyperplasia, thyroid diseases, hyperprolactinaemia;

7. Known allergy to DHEA;

8. Diabetic women on insulin, as insulin lessens the DHEA levels and might reduce the effectiveness of DHEA supplements.

\section{Recruitment and randomisation}

At the initial visit, women seeking IVF/ICSI treatment will undergo an ultrasound scan for AFC as well as an $\mathrm{AMH}$ blood test. If a woman fulfils the eligibility criteria, she will be invited to participate in this study. After written signed consent is obtained, participants will be randomised to receive oral capsules of either $75 \mathrm{mg}$ DHEA or placebo (once daily) for at least 12 weeks (1220 weeks) before starting ovarian stimulation and continued through stimulation until the day before egg collection (figure 1). The randomisation will be based on a computer-generated pseudo-random code using random permuted blocks of randomly varying size, created by the University of Nottingham Clinical Trials Unit (CTU). The randomisation will be stratified by age. At randomisation, the computer will then issue a trial number, which will be the unique identifier for the trial participant. The corresponding treatment allocation will be transmitted to the unblinded pharmacy staff, who will dispense the allocated treatment (trial medication or placebo) to the participant.

The randomisation code will be maintained by the CTU. The pharmacy will have the list of participants with their allocated treatment. The code will be broken only if any participants develop a serious adverse event, in which case the chief investigator will be notified.

\section{Primary objectives}

This study aims to evaluate the hypothesis that DHEA supplementation for at least 12 weeks prior to and during controlled ovarian hyperstimulation in patients predicted to be poor responders increases the oocyte quantity (number of oocytes retrieved). The feasibility of conducting a large trial is also to be assessed by evaluating the recruitment rates and compliance of the recruited participants with DHEA/placebo intake and follow-up rates.

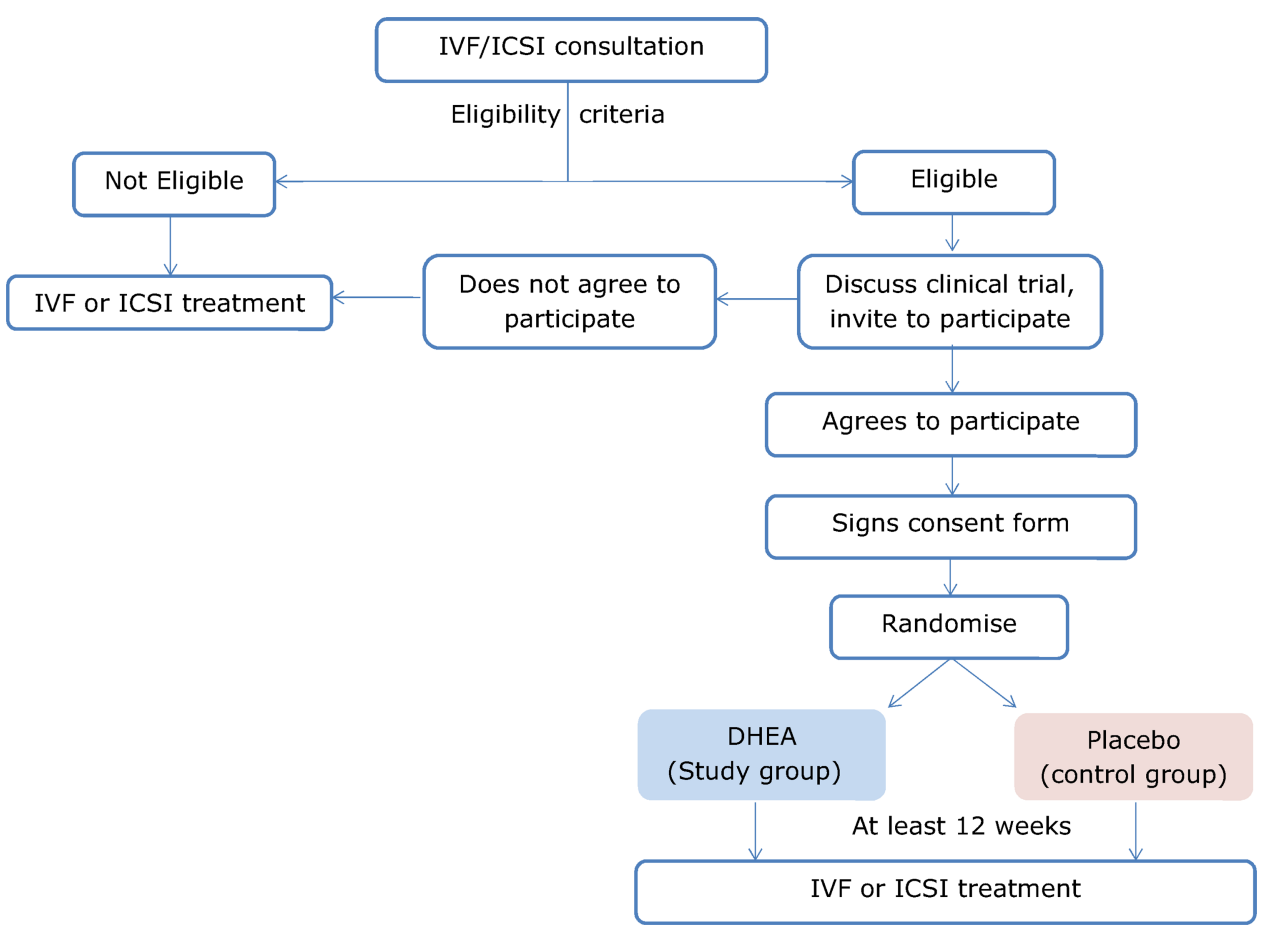

Figure 1 Participant flow diagram (IVF, in vitro fertilization; ICSI, intracytoplasmic sperm injection; DHEA, dehydroepiandrosterone). 


\section{Secondary objectives}

Oocyte quality will be determined by expressions of established molecular markers. The expression levels of various cumulus markers including gonadotropin receptors, pentraxin 3 , cyclooxygenase 2 , hyaluronan synthase 2, bone morphogenetic protein (BMP) antagonistgremlin and epidermal growth factor-like signalling molecules will be measured to define oocyte developmental competence using real-time PCR. Nutritional fingerprinting by measuring glucose, pyruvate and lactate utilisation as the energy consumption from the oocyte culture media will be evaluated to assess the developmental competence of the fertilised oocytes. Finally, we will also be assessing aneuploidy rates in immature oocytes to see any beneficial effect of DHEA on oocyte quality. Biochemical (positive urine human chorionic gonadotropin (hCG) at 2 weeks following embryo transfer) and clinical (viable pregnancy on ultrasound at 5 weeks following embryo transfer) pregnancy rates will also be reported as secondary outcomes. However, the present study is underpowered to measure plausible effect sizes.

\section{Procedure}

All participants will undergo a standard long downregulation protocol (figure 2) starting with the GnRH agonist, either nafarelin (Synarel; Pharmacia, UK) or buserelin (Aventis Pharma, Kent, UK), 7 days earlier than the expected first date of the next menstrual cycle. Pituitary downregulation will be confirmed by both a transvaginal ultrasound scan (quiescent ovaries with follicles $<10 \mathrm{~mm}$ diameter, endometrium $\leq 5 \mathrm{~mm}$ in thickness) and serum oestradiol $(<200 \mathrm{pmol} / \mathrm{L})$ on day 2 after the first day of the menstruation period. A sample of serum from the downregulation blood sample will be stored for serum DHEA and AMH estimation. On confirmation of downregulation, ovarian stimulation will be started with Human Menopausal Gonadotropin (HMG, Menopur, Ferring, UK) $300 \mathrm{IU}$ daily by subcutaneous injection. From day 8 of stimulation, participants will be monitored for their ovarian response using transvaginal ultrasound and serum oestradiol levels. Once they meet the criteria for oocyte retrieval $(\geq 3$ leading follicles are $\geq 17 \mathrm{~mm}$ ), $10000 \mathrm{IU}$ of hCG, Pregnyl (Organon Laboratories Ltd, Cambs, UK) will be administered subcutaneously to trigger oocyte maturation. The trial intervention, gonadotropin injections and $\mathrm{GnRH}$ agonist will be continued until hCG administration. Thirty-six hours following HCG administration, oocyte retrieval from ovarian follicles will be performed (figure 2).

Extra blood samples of serum DHEA and AMH concentrations will be collected on the day of egg collection. Conventional insemination (IVF) or ICSI treatment will be appropriately selected for individual patients by embryologists depending on the partner's semen analysis and previous treatment history. Prior to ICSI, the oocytes will be denuded prior to sperm injection according to standard clinical practices. Expression levels of a panel of nine cumulus markers of oocyte competence as aforementioned will be quantified by real-time PCR in cumulus cells removed from one cumulus oocyte complex (COC)/patient. The metaphase II oocytes from these selected COCs will be inseminated according to standard clinical practices. We estimate that the ICSI treatment rate will be approximately $50 \%$ of total patients, and thus will be able to have cumulus markers data from about 30 patients. Microdrop culture will be used to track individual zygotes throughout embryo development in vitro, until transfer on day 2, 3 to 5 or cryopreservation. Oocyte developmental competence will then be assessed indirectly by measuring glucose $(\mathrm{G})$, pyruvate $(\mathrm{P})$ and lactate $(\mathrm{L})$ utilisation from the culture media as the energy consumption by the oocyte from the medium ('nutritional finger printing') will vary depending on its ability to develop to the blastocyst stage. The fluid within the follicle from which the above oocytes are collected will be stored and analysed for

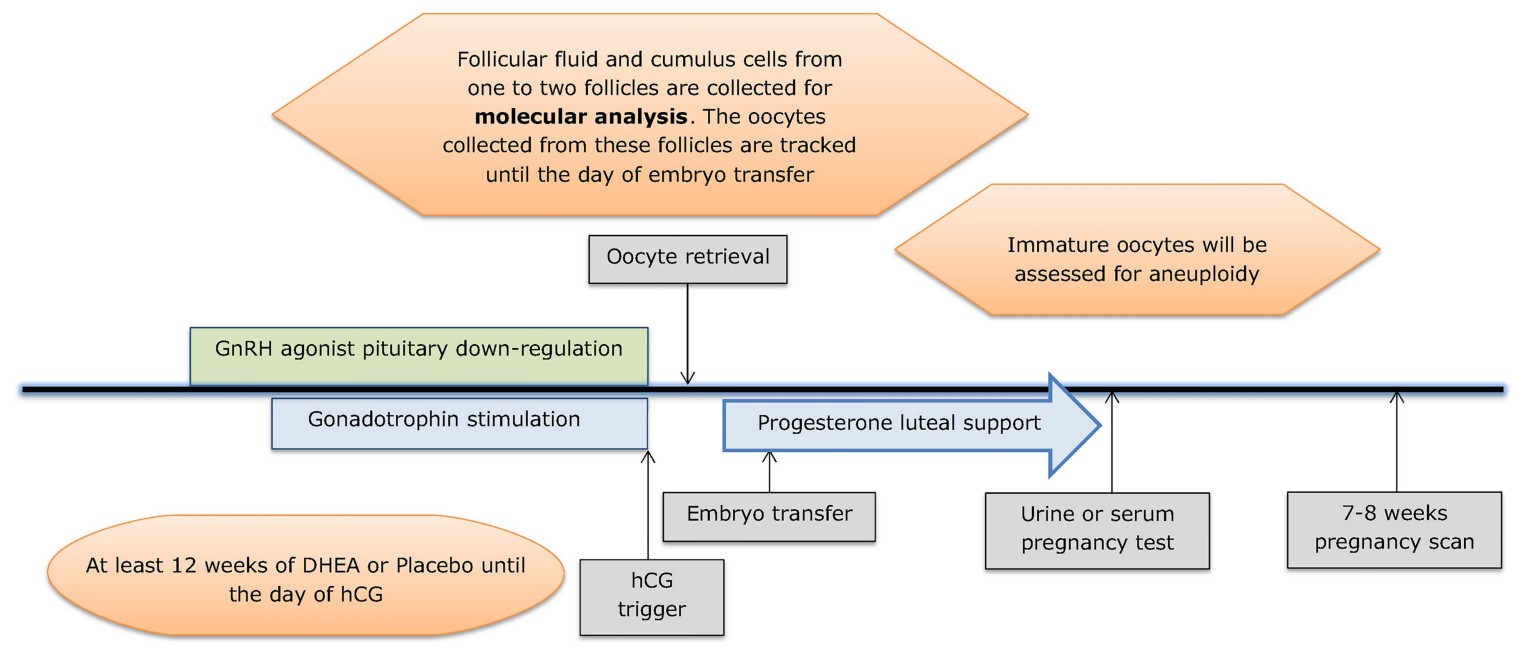

Figure 2 IVF/ICSI treatment protocol used in the study (IVF, in vitro fertilization; ICSI, intracytoplasmic sperm injection; DHEA, dehydroepiandrosterone; hCG, human chorionic gonadotropin). 
DHEA levels. Chromosomal assessment of the immature oocytes recovered at denudation while doing ICSI will be performed by using comparative genomic hybridisation. Participants will be reassured that the oocytes that are utilised for the research would have no further role in their treatment; therefore, taking part in the study would not affect their treatment outcome.

Embryo transfer is performed 2, 3 or 5 days following the day of egg collection, based on the number and quality of embryos available. One or two embryos will be transferred depending on the embryo quality and couple preference. The maximum number of embryos transferred will be two as per the Human Fertilisation and Embryology Authority (HFEA) regulations. The couples will be advised by the embryologists regarding blastocyst culture and transfer if there are $\geq 4$ fertilised oocytes at fertilisation check. Couples will be advised for single embryo transfer if top quality blastocysts are available for transfer. All women undergoing IVF treatment will be advised to self-administer progesterone pessaries (Cyclogest; Alpharma, UK) $400 \mathrm{mg}$ twice daily starting on the second day after egg collection until the day of the pregnancy test, which will then be continued until 8 weeks of pregnancy if the treatment is successful. A urine pregnancy test will be performed approximately 14 days from the day of embryo transfer. All women having IVF/ICSI treatment will have an early pregnancy scan at around 7 weeks of gestation (clinical pregnancy; figure 2). All the stimulation and outcome data will be collected and recorded on to the database. Side effects reported by women will be recorded.

The project will be performed over a period of 2 years and the last follow-up visit of the participants will be the day of the first early pregnancy scans. While we are planning to do the molecular analysis and chromosomal analysis during the last phase of the trial, we will prospectively collect the baseline and clinical outcome data as participants go through the trial and undergo IVF treatment.

\section{Statistical analysis}

The trial is designed to test the 'proof of principle' that DHEA priming improves the ovarian response to superovulation. The primary outcome is the total number of oocytes retrieved. Only the first cycle of treatment following randomisation will be analysed. If treatment cycles are cancelled during the stimulation phase due to poor ovarian response or if no eggs are retrieved at egg collection, the number of eggs collected will be recorded as zero. All participants will be advised to use a barrier contraception while taking trial medications. However, if participants spontaneously conceive during DHEA/placebo treatment, for the primary analysis we will assign the number of the patient's oocyte count with the highest observed number in the rest of the study while this patient will be included into the positive pregnancy outcome group for secondary (pregnancy) outcome analysis. The secondary outcome will include
(1) biochemical/clinical pregnancy rates, (2) live birth rates, (3) relative quotients of nine cumulus gene expressions using real-time PCR, (4) rates of glucose, pyruvate and lactate consumption, (5) rates of aneuploidy in immature oocytes, (6) serum oestradiol on hCG day and (7) serum AMH on the sample collected at egg collection (after treatment).

As this is a pilot study, we estimated the sample size based on the number of participants that we could recruit over a period of 18 months. We anticipate approximately 600 women undergoing IVF/ICSI treatment at NURTURE over an 18-month period. Considering $20 \%$ as our predicted poor responders based on the inclusion criteria and $50 \%$ recruitment rate, we will be able to recruit 60 participant during the study period. We anticipate that the size of the effect on the primary outcome based on the data generated from this trial will enable us to estimate the sample size for a large multicentre trial.

Statistical analysis will be performed by using the Statistical Package for the Social Sciences (SPSS, Chicago, Illinois, USA). Assuming that the oocyte numbers in a study population are usually not normally distributed, the difference in the primary outcome between groups will be tested using the Mann-Whitney $\mathrm{U}$ test. A two-tailed $\mathrm{p}$ value of $<0.05$ will be taken to indicate statistical significance. Analyses will be performed between the study and control participants who have complied with the trial as per protocol and not just on the intention to treat basis.

\section{Ethics and dissemination}

The side effects associated with the proposed dose of DHEA are rare. The published cohort studies and the RCT have not reported any significant adverse or androgenic side effects. ${ }^{20-23}{ }^{26}$ However, the potential side effects are related to androgenic effects, and these include acne, facial hair growth and rarely a deepening of the voice. ${ }^{35}$ If women report any of these side effects, they will be advised to discontinue the trial medication. The notes of all pregnant participants will be retrospectively reviewed to ensure the normality of the children born.

This study has received appropriate approval from the NHS Research Ethics Committee (East Midlands-Derby1 ethics committee; Ref number NRES 12/EM/0002), the Medicines and Healthcare products Regulatory Agency (MHRA) for use of investigational medicinal products, and the Nottingham University Hospitals Trust Research and Development department. It is being conducted in accordance with the good clinical practice (GCP) principles, the ethical principles that are in agreement with the Declaration of Helsinki 1996, the Medicines for Human Use Regulations, Statutory Instrument 2004, and all involved UK laws and regulations. All participants shall provide written informed consent before being randomised into allocated treatment groups. The 
participants will be informed which treatment they received after the trial is completed.

We are planning to disseminate the study through peer-reviewed journal publications and scientific conferences. The data that will be produced from this research will have the potential to influence clinical practice in fertility clinics worldwide. If the planned intervention is found to be effective, it provides a low cost, safe and easily administrable therapy for ovarian ageing. Furthermore, the overall results will give us more appreciation in understanding the underlying mechanisms of how DHEA combats reproductive ageing. This provides a mechanistic framework for translational research on mechanisms of ovarian ageing and drug interventions to slow down the ovarian ageing process and subsequent adverse consequences.

Acknowledgements The authors would like to thank Professor Jim Thornton for his help with the critical review and editing of the manuscript.

Collaborators Nicholas Raine-Fenning.

Contributors KJ, AN, WEM and BKC were responsible for the conception, design and analytical plan. Critical evaluation was performed by all authors. $\mathrm{KJ}, \mathrm{AN}$ and BKC were responsible for writing the first draft and the final manuscript was read and approved by all authors.

Funding This work has been supported by the University of Nottingham through the Early Career Research and Knowledge Transfer scheme and Nottingham University Hospital (NUH) Charity; Project code: A2RHD6.

Competing interests None.

Patient consent Obtained.

Ethics approval NHS Research Ethics Committee (Ref number NRES:12/EM/ 0002).

Provenance and peer review Not commissioned; externally peer reviewed.

Open Access This is an Open Access article distributed in accordance with the Creative Commons Attribution Non Commercial (CC BY-NC 4.0) license, which permits others to distribute, remix, adapt, build upon this work noncommercially, and license their derivative works on different terms, provided the original work is properly cited and the use is non-commercial. See: http:// creativecommons.org/licenses/by-nc/4.0/

\section{REFERENCES}

1. Broekmans FJ, Soules MR, Fauser BC. Ovarian aging: mechanisms and clinical consequences. Endocr Rev 2009;30:465-93.

2. Office for National Statistics. Chapter 2: Household and families. In: Self A and Zealey L, Social Trends No 38. Palgrave Macmillan, Hampshire, 2008:15-28.

3. Broekmans FJ, Kwee J, Hendriks DJ, et al. A systematic review of tests predicting ovarian reserve and IVF outcome. Hum Reprod Update 2006;12:685-718.

4. Broekmans FJ, Faddy MJ, Scheffer G, et al. Antral follicle counts are related to age at natural fertility loss and age at menopause. Menopause 2004;11:607-14.

5. Santoro N, Isaac B, Neal-Perry G, et al. Impaired folliculogenesis and ovulation in older reproductive aged women. J Clin Endocrinol Metab 2003;88:5502-9.

6. Soules MR, Sherman S, Parrott E, et al. Executive summary: Stages of Reproductive Aging Workshop (STRAW). Fertil Steril 2001;76:874-8.

7. Templeton A, Morris JK, Parslow W. Factors that affect outcome of in-vitro fertilisation treatment. Lancet 1996;348:1402-6.

8. Menken J, Trussell J, Larsen U. Age and infertility. Science 1986;233:1389-94.

9. Trout SW, Seifer DB. Do women with unexplained recurrent pregnancy loss have higher day 3 serum FSH and estradiol values? Fertil Steril 2000;74:335-7.
10. Gleicher N, Weghofer A, Barad DH. Defining ovarian reserve to better understand ovarian aging. Reprod Biol Endocrinol 2011; 9:23.

11. Freeman SB, Yang Q, Allran K, et al. Women with a reduced ovarian complement may have an increased risk for a child with Down syndrome. Am J Hum Genet 2000;66:1680-3.

12. Warburton D. Biological aging and the etiology of aneuploidy. Cytogenet Genome Res 2005;111:266-72.

13. Hodges CA, llagan A, Jennings D, et al. Experimental evidence that changes in oocyte growth influence meiotic chromosome segregation. Hum Reprod 2002;17:1171-80.

14. Nejat EJ, Chervenak JL. The continuum of ovarian aging and clinicopathologies associated with the menopausal transition. Maturitas 2010;66:187-90.

15. Traub ML, Santoro N. Reproductive aging and its consequences for general health. Ann N Y Acad Sci 2010;1204:179-87.

16. Fitzpatrick LA, Litin SC, Bell MR. The Women's Health Initiative: a heart-to-HRT conversation. Mayo Clin Proc 2000;75:559-61.

17. Li H, Simpson ER, Liu JP. Oestrogen, telomerase, ovarian ageing and cancer. Clin Exp Pharmacol Physiol 2010;37:78-82.

18. Hansen KR, Knowlton NS, Thyer AC, et al. A new model of reproductive aging: the decline in ovarian non-growing follicle number from birth to menopause. Hum Reprod 2008;23:699-708.

19. de Bruin JP, Bovenhuis $\mathrm{H}$, van Noord PA, et al. The role of genetic factors in age at natural menopause. Hum Reprod 2001;16:2014-18

20. Casson PR, Lindsay MS, Pisarska MD, et al. Dehydroepiandrosterone supplementation augments ovarian stimulation in poor responders: a case series. Hum Reprod 2000;15:2129-32.

21. Gleicher N, Weghofer A, Barad DH. Improvement in diminished ovarian reserve after dehydroepiandrosterone supplementation. Reprod Biomed Online 2010;21:360-5.

22. Barad D, Gleicher N. Effect of dehydroepiandrosterone on oocyte and embryo yields, embryo grade and cell number in IVF. Hum Reprod 2006;21:2845-9.

23. Barad D, Brill H, Gleicher N. Update on the use of dehydroepiandrosterone supplementation among women with diminished ovarian function. J Assist Reprod Genet 2007;24:629-34.

24. Barad DH, Gleicher N. Increased oocyte production after treatment with dehydroepiandrosterone. Fertil Steril 2005;84:756.

25. Sönmezer M, Ozmen B, Cil AP, et al. Dehydroepiandrosterone supplementation improves ovarian response and cycle outcome in poor responders. Reprod Biomed Online 2009;19:508-13.

26. Wiser A, Gonen O, Ghetler Y, et al. Addition of dehydroepiandrosterone (DHEA) for poor-responder patients before and during IVF treatment improves the pregnancy rate: a randomized prospective study. Hum Reprod 2010;25:2496-500.

27. Gleicher N, Barad DH. Dehydroepiandrosterone (DHEA) supplementation in diminished ovarian reserve (DOR). Reprod Biol Endocrinol 2011:9:67.

28. Yeung TW, Chai J, Li RH, et al. A randomized, controlled, pilot trial on the effect of dehydroepiandrosterone on ovarian response markers, ovarian response, and in vitro fertilization outcomes in poor responders. Fertil Steril 2014;102:108-15.e1.

29. Kara M, Aydin T, Aran T, et al. Does dehydroepiandrosterone supplementation really affect IVF-ICSI outcome in women with poor ovarian reserve? Eur J Obstet Gynecol Reprod Biol 2014;173:63-5.

30. Narkwichean A, Maalouf W, Campbell BK, et al. Efficacy of dehydroepiandrosterone to improve ovarian response in women with diminished ovarian reserve: a meta-analysis. Reprod Biol Endocrinol 2013;11:44.

31. Jayaprakasan K, Campbell B, Hopkisson J, et al. A prospective, comparative analysis of anti-Mullerian hormone, inhibin-B, and three-dimensional ultrasound determinants of ovarian reserve in the prediction of poor response to controlled ovarian stimulation. Fertil Steril 2010;93:855-64.

32. Jayaprakasan $\mathrm{K}$, Chan $\mathrm{Y}$, Islam $\mathrm{R}$, et al. Prediction of in vitro fertilization outcome at different antral follicle count thresholds in a prospective cohort of 1,012 women. Fertil Steril 2012:98:657-63.

33. Jayaprakasan $\mathrm{K}$, Al-Hasie $\mathrm{H}$, Jayaprakasan $\mathrm{R}$, et al. The three-dimensional ultrasonographic ovarian vascularity of women developing poor ovarian response during assisted reproduction treatment and its predictive value. Fertil Steril 2009;92:1862-9.

34. Nelson SM, Yates RW, Lyall H, et al. Anti-Mullerian hormone-based approach to controlled ovarian stimulation for assisted conception. Hum Reprod 2009;24:867-75.

35. Panjari M, Davis SR. DHEA therapy for women: effect on sexual function and wellbeing. Hum Reprod Update 2007;13:239-48. 